\title{
Avanços e retrocessos no estabelecimento de indicadores de saúde bucal 2007 a 2019 no Brasil
}

\section{Advances and returns in the establishment of oral health indicators 2007 to 2019 in Brazil}

\section{Avances y devoluciones en el establecimiento de indicadores de salud oral 2007 a 2019 en Brasil}

Ruann Oswaldo Carvalho da Silva Guilherme Fernandes Graziani ${ }^{2}$ Rafael Gomes Ditterich ${ }^{3}$

RESUMO: O Ministério da Saúde durante as últimas décadas vem recomendando o uso de indicadores para a avaliação e a monitoração da atenção em saúde bucal, com o propósito de estimular gestores do sistema de saúde a incorporarem nas suas práticas na organização e desempenho dos serviços de saúde. O objetivo do presente artigo foi analisar a evolução dos indicadores de saúde bucal do Sistema Único de Saúde (SUS). Para isso, foi realizada pesquisa documental com base nas resoluções, portarias e manuais governamentais editados no período analisado de 2007 a 2018. Constatou-se que mudanças foram identificadas ao longo dos anos, caracterizadas pela inclusão e exclusão de indicadores de saúde bucal e por uma redução drástica nos últimos 2 anos, o que pode comprometer realmente na avaliação em saúde bucal nos municípios brasileiros e na qualidade dos serviços ofertados à população.

Palavras-chaves: Indicadores de serviços; saúde bucal; gestão em saúde

\begin{abstract}
The Ministry of Health over the last decades has been recommending the use of indicators for the evaluation and monitoring of oral health care, with the purpose of encouraging health system managers to incorporate in their practices in the organization and performance of health services. The aim of this article was to analyze the evolution of oral health indicators of the Unified Health System (SUS). For this, a documentary research was carried out based on

1 Doutorando do Programa de Pós-Graduação em Políticas Públicas, Universidade Federal do Paraná

2 Mestre em Políticas Públicas, Programa de Pós-Graduação em Políticas Públicas, Universidade Federal do Paraná

3 Professor do Departamento de Saúde Coletiva, Universidade Federal do Paraná
\end{abstract}

ISSN 1982-8829 Tempus, actas de saúde colet, Brasília, 14(1), 65-75, mar, 2020. Epub Mai/2020 
the resolutions, ordinances and government manuals issued in the period analyzed from 2007 to 2018. It was found that changes were identified over the years, characterized by the inclusion and exclusion of oral health indicators and by a drastic reduction in the last 2 years, which can really compromise oral health assessment in Brazilian municipalities and the quality of services offered to the population.

Keywords: Service indicators; oral health; health management

RESUMEN: El Ministerio de Saludenlas últimas décadas ha recomendado el uso de indicadores para laevaluación y elmonitoreo de laatención de lasalud bucal, conel propósito de alentar a los administradores del sistema de salud a incorporar sus prácticasenlaorganización y eldesempeño de losservicios de salud. El objetivo de este artículo fueanalizarlaevolución de los indicadores de salud bucal del Sistema Único de Salud (SUS). Con este fin, se realizó una investigación documental basadaenlasresoluciones, ordenanzas y manualesgubernamentales emitidos de 2007 a 2018. Se descubrió que loscambios se identificaron a lo largo de losaños, caracterizados por lainclusión y exclusión de indicadores de salud oral y por unreducción drástica enlos últimos 2 años, lo que realmente puede comprometer laevaluación de lasalud bucal enlosmunicipiosbrasileños y lacalidad de losserviciosofrecidos a lapoblación.

Palabras clave: Indicadores de servicio; salud bucal; gestión de lasalud

\section{INTRODUÇÃO}

A efetivação dos princípios do Sistema Único de Saúde (SUS) depende de um processo de avaliação que precisa ser constante e estar subordinado aos objetivos e metas do sistema e, entre outros parâmetros, faz-se necessário a análise de indicadores adequados à realidade específica de cada situação ${ }^{1}$.

A avaliação em saúde tem como propósito fundamental dar suporte ao processo decisório e, por isso, deve subsidiar a identificação de problemas e a reorientação de ações e serviços desenvolvidos, avaliar a incorporação de novas práticas sanitárias na rotina de profissionais e mensurar o impacto das ações implementadas pelos serviços e programas sobre o estado de saúde da população².

A institucionalização da avaliação dos serviços de saúde constitui um desafio para todos os níveis de gestão do SUS $^{3-5}$ e vem sendo discutida pela sociedade brasileira ${ }^{6-8}$. Ela objetiva contribuir para a tomada de decisões, planejamento, programação e organização do serviço, tendo o cuidado de se levar em conta os sujeitos envolvidos na prática do serviço ${ }^{4}$.

Os indicadores de saúde são essenciais para o planejamento, gestão e avaliação em saúde. Apoiam a elaboração de políticas públicas e o estabelecimento de prioridades para atender às necessidades da população? .

Nas últimas décadas, o Ministério da Saúde instituiu o sistema de pactos interfederativos com 
o objetivo de incentivar os gestores do SUS a incorporar o monitoramento e avaliação de ações em sua prática, bem como possibilitar o acompanhamento do desempenho de serviços de saúde ${ }^{10}$.

Os pactos foram projetados para se tornarem uma ferramenta para negociação pelas três entidades federadas, e estabelecer as metas a serem alcançadas e os indicadores de saúde previamente recomendados e acordados. Eles contêm uma lista mínima de indicadores - incluindo aqueles para gestão da saúde - a ser adotada pelos municípios e estados, bem como as orientações técnicas para o cálculo dos indicadores, e os prazos e fluxos do processo de construção do pacto no nível nacional ${ }^{10}$.

A importância da saúde bucal tem sido crescente no cenário nacional e o processo de descentralização favoreceu aos gestores o planejamento das ações conforme a realidade local, requerendo destes o conhecimento da situação de saúde e do desempenho do sistema para tomada de decisão em saúde ${ }^{11}$.

Em geral, tanto os indicadores quanto os fatores que podem influenciar seu uso na prática de serviço público ainda são bastante desconhecidos, apesar da disponibilidade de uma grande quantidade de informações sobre sua importância e propósito. Em um estudo foi verificado, as seguintes dificuldades e limitações no uso de indicadores de saúde foram apontadas pelos gestores: lentidão, falta de integração dos sistemas de saúde, e falta de treinamento para gerentes sobre como operar o sistema ${ }^{12}$.

Neste contexto, questiona-se quais são os indicadores em saúde bucal que estão disponíveis para a gestão em saúde, para que de fato possa avaliar as políticas públicas implementadas e as ações propostas, bem como o desempenho do sistema e os resultados alcançados. São escassos os estudos acerca destes indicadores, incluindo os dados necessários para o seu cálculo e a sua fonte. Portanto, o objetivo deste artigo foi analisar o desenvolvimento e estabelecimento de indicadores de saúde bucal propostos pelo Ministério da Saúde a partir de 2007.

\section{PERCURSO METODOLÓGICO}

O presente estudo é de abordagem qualitativa, com desenho exploratório descritivo, utilizandoa técnica da análise documental. Esta técnica permitirá descrever e representar o conteúdo,registrando as informações contidas nos documentos originais ${ }^{13}$.

Analisou-se o desenvolvimento e estabelecimento de indicadores de saúde bucal propostos pelo Ministério da Saúde a partir de 2007, partindo do Pacto pela Saúde ao Contrato Organizativo de Ação Pública (COAP).

Ométodo de análise de conteúdo ${ }^{14}$ foi aplicado para análisedos dados, que procura extrairatravés das palavras sentido dos textos, concepções de mundo, interesses de classe, motivações eexpectativas, permitindo ao autor fazer inferências correlacionadas a luz das concepções teóricas ${ }^{15}$. 
Todos os documentos analisados são de domínio público, dispensando a submissão ao Comitêde Ética em Pesquisa.

\section{RESULTADOS E DISCUSSÃO}

O levantamento epidemiológico nacional de saúde bucal realizado pelo Ministério da Saúde do Brasil em $2003^{16}$ produziu subsídios para o planejamento e avaliação de ações de saúde bucal. Ficando assim, demonstrado a persistência de desigualdades regionais das condições de saúde bucal da população brasileira, o que exige, além de ações sobre os determinantes da incidência das doenças e agravos bucais, serviços destinados a minimizar suas consequências.

Esse levantamento revelou que o índice de acometimento da cárie dentária na dentição permanente (CPO-D) atingiu a média de 2,8 dentes aos 12 anos de idade, eleva-se a 6,2 na população da faixa etária entre 15 a 19 anos. Na faixa etária adulta (35 a 44 anos) e entre os idosos (65 a 74 anos) atinge níveis alarmantes com valores médios de 20,1 e 27,8 respectivamente. As desordens oclusais, nas suas formas severa, muito severa e incapacitante, atingem 36,6\% dos escolares de 12 anos de idade. Mais de $28 \%$ dos adultos brasileiros apresentam nenhum dente funcional em pelo menos uma arcada e 15\% deles não possuem prótese total. Entre os idosos a situação ainda é mais grave, pois $75 \%$ (não) apresentam nenhum dente funcional e $36 \%$ deles não possuem prótese total modificada ${ }^{16}$.

Contribuindo para esta compreensão, encontram-se também a falta de um referencial teórico que permita a descrição e avaliação sistemática dos vários indicadores para a gestão da saúde bucal, publicados nas diretrizes governamentais para os diferentes serviços de saúde no Brasil, bem como a indisponibilidade de dados necessários para calcular esses indicadores e informações sobre sua fonte.

Um estudo conduzido por Bordin e Fadel (2012) ${ }^{17}$ analisou a evolução temporal dos indicadores de saúde bucal de 2008 a 2010 nas bases de dados do Departamento de Informática do SUS (DATASUS), e apontou que, desde então, as diretrizes do governo foram instituídas para orientar a construção do pacto, e fornecer uma lista de indicadores a serem acordados pelos gestores, sendo escassas as propostas apresentadas para indicadores de saúde bucal.

\section{INDICADORES DE SAÚDE BUCAL: PARTINDO DO PACTO PELA SAÚDE AO CONTRATO ORGANIZATIVO DE AÇÃO PÚBLICA (COAP)}

Em 2006, a Portaria n. 493, de 13/03/2006, estabeleceu os principais indicadores do Pacto de Atenção Básica, que passam a ser calculados a partir das bases nacionais dos diversos sistemas do Ministério da Saúde ${ }^{18}$. Desde então, esses indicadores têm sido utilizados na gestão como instrumento na tomada de decisão, para avaliar ações e subsidiar o seu redirecionamento ${ }^{19}$. Este ano correspondeu a uma fase de maior progresso na evolução dos indicadores em saúde bucal, com a reintegração do indicador medidas preventivas e inclusão de novos e importantes indicadores 
relacionados aos cuidados básicos e especializados.

Com a publicação do Pacto pela Saúde, assinado pelos três níveis de gestão, este estabeleceu novas formas de relacionamento e negociação entre os gestores do SUS, compreendendo três componentes: o Pacto pela Vida, o Pacto em Defesa do SUS e o pacto pela gestão. A regulamentação do Pacto pela Saúde envolveu a implementação diretrizes operacionais para o processo de gestão do SUS, e para a transição e monitoramento dos Pactos pela Vida e Gestão, unificando assim os processos de acordo em relação aos indicadores e alvos. No entanto, a unificação completa desses processos ocorreu apenas em $2007^{20}$.

No período entre 2007 e 2011 (Tabela 1), foram feitas mudanças nos indicadores de saúde bucal, com exceção de $2010^{21}$, quando novos indicadores foram incorporados e outros foram excluídos. Em 2009, a Portaria n. 2.669 de 3 de novembro de $2009^{22}$ foi emitida com a lista de indicadores do Pacto pela Saúde para o biênio 2010-2011, nas várias áreas prioritárias; no entanto, indicadores de saúde bucal para 2010 não foram incluídos nesta lista, com a emissão da Portaria n. 3840 de 12 de dezembro de $2010^{23}$, na qual esta portaria estabeleceu as diretrizes, instruções e prazos para o processo de ajuste de metas para 2011.

TABELA 1 - Evolução dos indicadores de saúde bucal no pacto pela saúde

\begin{tabular}{c|c|c|c|c}
\hline Indicadores & 2007 & 2008 & 2009 & 2011 \\
\hline Cobertura de primeira consulta odontológica programática & $\mathrm{X}$ & $\mathrm{X}$ & $\mathrm{X}$ & \\
\hline Cobertura da ação coletiva escovação dental supervisionada & $\mathrm{X}$ & $\mathrm{X}$ & $\mathrm{X}$ & $\mathrm{X}$ \\
\hline Média de procedimentos odontológicos básicos individuais & $\mathrm{X}$ & & & \\
\hline $\begin{array}{c}\text { Cobertura de implantação de Equipe de Saúde Bucal } \\
\text { Proporção de procedimentos odontológicos especializados } \\
\text { em relação às ações odontológicas individuais }\end{array}$ & & $\mathrm{X}$ & $\mathrm{X}$ & \\
\hline $\begin{array}{c}\text { Cobertura populacional estimada das Equipes de Saúde } \\
\text { Bucal da Estratégia de Saúde da Família }\end{array}$ & & & & \\
\hline
\end{tabular}

Fonte: Portarias GM/MS No. 91/07; No. 325/08; $\mathrm{N}^{\circ} .48 / 09, \mathrm{~N}^{\circ} .3840 / 10$.

Disponível em: http://portal2.saude.gov.br/saudelegis/leg_norma_pesq_consulta.cfm

Uma proposta de novo pacto foi feita em junho de 2011, quando o Decreto n. 7.508 de 28 de junho de $2011^{24}$ foi emitido definindo novos critérios, ferramentas de construção de pactos e ferramentas de monitoramento utilizado pelas entidades federadas, por meio do Contrato Organizativo de Ação Pública (COAP). A publicação da Portaria n. 1.580 de julho de $2012^{25}$ liberou os administradores da obrigação de cumprir o Pacto pela Saúde ou assinar o Termo de Adesão Compromisso de Gestão ("Termo de Compromisso de Gestão"), e estabeleceu o Contrato Organizativo de Ação Pública (COAP) como a ferramenta federativa apropriada para formalizar relações interfederativas. Os dois indicadores de saúde bucal utilizados na transição do pacto pela Saúde ao COAP em 2012 (Tabela 2), visam garantir acesso equitativo e oportuno da população a serviços de qualidade. 
Enquanto aguarda a finalização do processo de reorientação do pacto, a Resolução do CIT n. 04 foi publicada em agosto de 2012, que previa o acordo tripartido sobre as regras relativas às responsabilidades sanitárias no âmbito do SUS, sendo seu objetivo realizar a transição dos processos operacionais do Pacto pela Saúde para o COAP.

Após o período de transição, o COAP 2012 inovou incluindo indicadores relacionados ao processo de educação permanente profissionais de saúde, com o objetivo de contribuir para a formação, alocação, qualificação e valorização adequadas do SUS profissionais, além de promover a democratização do trabalho relações ${ }^{18,26}$.

Em 2012, o Ministério da Saúde publicou um manual com as metas e indicadores que compõem o COAP, e acrescentou um conjunto de diferentes indicadores para a lista desse ano ${ }^{27}$. Os indicadores do Pacto pela Saúde foram mantidos e dois novos e diferentes indicadores foram incorporados abordando a saúde bucal. A Resolução n. 5 de 2013 definiu três indicadores de saúde bucal para triênio 2013-2015 (Tabela 2). Embora esta resolução tenha reduzido o número de indicadores em relação a 2012, manteve indicadores importantes para o monitoramento de ações. No entanto, a emissão da Resolução n. 2 de 2016 excluiu todos os anteriormente indicadores propostos, e manteve apenas um: "Proporção de exodontia em relação aos procedimentos"18. Em 2016, é publicada a resolução n. 8/2016 a qual estabelece uma nova mudança, e mantem somente o indicador "Cobertura populacional estimada de saúde bucal na Atenção Básica” que já existia na pactuação de 2013-15.

TABELA 2 - Evolução dos indicadores de saúde bucal no contrato organizativo da ação pública da saúde (COAP).

\begin{tabular}{c|c|c|c|c}
\hline Indicadores & $\mathbf{2 0 1 2}$ & $\begin{array}{c}\mathbf{2 0 1 3}- \\
\mathbf{2 0 1 5}\end{array}$ & $\mathbf{2 0 1 6}$ & $\begin{array}{c}\mathbf{2 0 1 7 -} \\
\mathbf{2 0 2 1}\end{array}$ \\
\hline Média da ação coletiva de escovação dental supervisionada & $\mathrm{X}$ & $\mathrm{X}$ & & \\
\hline $\begin{array}{c}\text { Cobertura populacional estimada de saúde bucal na Atenção } \\
\text { Básica }\end{array}$ & $\mathrm{X}$ & $\mathrm{X}$ & & $\mathrm{X}$ \\
\hline Proporção de exodontia em relação aos procedimentos & & $\mathrm{X}$ & $\mathrm{X}$ & \\
\hline
\end{tabular}

Fonte: CIT Resoluções No. 4/12; No. 5/13, No. 2/16; No. 8/16.

Disponível em: http://www.conass.org.br/resolucoes-cit/

Ao analisar os indicadores de saúde bucal, França et al. (2018) ${ }^{26}$ argumentam que indicadores do Pacto pela Saúde em vigor no período 2007-2011 representaram uma proposta promissora de avaliação de serviços no campo saúde bucal e incluiu uma avaliação não apenas do impacto de ações realizadas pelos serviços nesta área, mas também no nível de acesso da população aos serviços oferecidos. No entanto, foi uma interrupção na proposição de indicadores de saúde bucal no Pacto pela Saúde para 2010, e os indicadores e metas para 2011 foram apresentados apenas no 
final de 2010.

No triênio 2013-2015, vários indicadores foram propostos. No entanto, ao contrário do esperado, houve uma gradual diminuição do número de indicadores de saúde bucal. Em 2016, o único indicador proposto para o monitoramento das ações nessa área foi o "Proporção de exodontias em relação aos procedimentos", similar à "Proporção de exodontias em relação aos procedimentos básicos", que fazia parte da lista de indicadores do Pacto de Atenção Básica no período de 2002 a 2006. Assim, apesar da contribuição potencial esperada dos indicadores estabelecidos no COAP, houve uma redução drástica no número de indicadores para a área de saúde bucal ${ }^{26}$.

No que se refere ao Programa Nacional de Melhoria do Acesso e da Qualidade da Atenção Básica (PMAQ-AB), três ciclos foram instituídos pelo Ministério da Saúde, sendo do primeiro em 2011, o segundo em 2013 e o último em 2015. Diferentes indicadores de saúde bucal foram identificados. Os dois primeiros ciclos contemplaram sete indicadores, no qual foram classificados em duas dimensões: desempenho e monitoramento. Os indicadores propostos, retratavam sobre: acesso e continuidade do cuidado, e resolutividade e oferta de serviços de saúde bucal. Já no último Ciclo do PMAQ-AB houve uma redução dos 7 indicadores para 2 indicadores até então utilizados, e incorporando mais um: Percentual de serviços ofertados pela Equipe de Saúde Bucal (Tabela 3).

TABELA 3 - Evolução dos indicadores de saúde bucal no programa nacional de melhoria do acesso e da qualidade da atenção básica (PMAQ-AB).

\begin{tabular}{|c|c|c|c|}
\hline Indicadores & $\mathbf{1}^{\mathrm{o}}$. ciclo & $2^{\circ}$. ciclo & $3^{\circ}$. ciclo \\
\hline Média da ação coletiva de escovação dental supervisionada & $\mathrm{X}$ & $\mathrm{X}$ & \\
\hline Cobertura de primeira consulta odontológica programática & $\mathrm{X}$ & $\mathrm{X}$ & $\mathrm{X}$ \\
\hline $\begin{array}{l}\text { Cobertura de } 1^{\mathrm{a}} \text { consulta de atendimento odontológico à } \\
\text { gestante }\end{array}$ & $\mathrm{X}$ & $\mathrm{X}$ & \\
\hline $\begin{array}{l}\text { Razão entre tratamentos concluídos e primeiras consultas } \\
\text { odontológicas programáticas }\end{array}$ & $X$ & $X$ & $X$ \\
\hline Média de instalações de próteses dentárias & $\mathrm{X}$ & $\mathrm{X}$ & \\
\hline $\begin{array}{l}\text { Média de atendimentos de urgência odontológica por } \\
\text { habitante }\end{array}$ & $X$ & $\mathrm{X}$ & \\
\hline Taxa de incidência de alterações da mucosa oral & $\mathrm{X}$ & $\mathrm{X}$ & \\
\hline Percentual de serviços ofertados pela Equipe de Saúde Bucal & & & $X$ \\
\hline
\end{tabular}

Fonte: Ficha de Qualificação dos Indicadores (2012) e Manual Instrutivo (2017).

Disponível em: http://dab.saude.gov.br/portaldab/ape_pmaq.php

Nos últimos 50 anos, tem sido observada no Brasil melhoria significativa nas estatísticas de saúde. Alguns autores ${ }^{28,29}$ afirmam que isto se deve não somente ao aumento do nível de renda e educação da população, mas também à melhoria e à disponibilidade dos serviços de saúde. 


\section{CONSIDERAÇÕES FINAIS}

Ao longo dos anos, o uso de indicadores permitiu analisar a quantidade e a qualidade dos serviços de saúde bucal prestados, e determinar se são ou não apropriados e suficientes para atender a demanda existente. Os indicadores também demonstram se as ações planejadas devem ser melhoradas e alteradas, e determinam se o nível de cumprimento dessas ações com as reais necessidades do SUS.

No entanto, a finalidade maior desse artigo não foi discutir se os indicadores em saúde bucal propostos pelo Ministério da Saúde são ideais ou não para mensurar a qualidade dos serviços e ações de saúde bucal no serviço público, mas comprovar que com o decorrer dos anos, mais especificamente nos últimos dois anos, houve uma redução drástica do número deles, o que pode comprometer a avaliação em saúde bucal nos municípios, já que os indicadores que verificavam acesso e qualidade nos diferentes ciclos de vida e o cuidado integral foram excluídos nos últimos documentos técnicos do Ministério da Saúde.

Portanto, recomenda-se que sejam estabelecidos diálogos com a participação de atores de diferentes áreas (gestão, controle social e academia, entre outros) para identificar e estabelecer uma lista de indicadores abrangendo aspectos inerentes ao planejamento e avaliações de ações no campo da saúde bucal.

\section{REFERÊNCIAS BIBLIOGRÁFICAS}

1. Campos GWS. Há pedras no meio do caminho do SUS! CienSaude Colet., v. 2, n. 2, p. 298 299, 2007.

2. Brasil. Ministério da Saúde. Caderno de atenção básica: Saúde Bucal. Brasília: MS, 2006.

3. Novaes HMD. Avaliação de programas, serviços e tecnologias em saúde Revista Saúde Pública, v. 34, n. 5, p. 547-59, 2000.

4. Teixeira CF. Formulação e implementação de políticas públicas saudáveis: desafios para o planejamento e gestão das ações de promoção da saúde nas cidades. Saúde e Sociedade v.13, n.1, p.37-46, jan-abr 2004.

5. Campos GWS. Há pedras no meio do caminho do SUS! CienSaude Colet., v. 2, n. 2, p. 298 299, 2007.

6. Hartz ZMA, Vieira-Da-Silva LM. (orgs.) a. Salvador: EdUFBA/Rio de Janeiro: Editora

Tempus, actas de saúde colet, Brasília, 14(1), 65-75, mar, 2020. Epub Mai/2020 ISSN 1982-8829 
Fiocruz; 2005.

7. Felisberto E. Da teoria à formulação de uma política nacional de avaliação em saúde: reabrindo o debate. Revista CienSaude Colet., v. 11, n. 3, p. 553-563. 2006.

8. Bodstein R. Atenção Básica na Agenda da Saúde. CienSaude Colet., v. 7, n. 3, p. 401-412, 2002 .

9. Tanaka OU,Tamaki EM. O papel da avaliação para a tomada de decisão na gestão dos serviços de saúde. Cien Saúde Colet., v. 17, n. 4, p. 821- 8. 2012.

10. Medina MG. Análise do pacto de indicadores da atenção básica, 2001 Brasília: Ministério da Saúde; 2002. Disponível em: http://bvsms.saude.gov.br/bvs/ publicações/analise_pacto.PDF

11. Lessa CF,Vettore MV. Gestão da Atenção Básica em Saúde Bucal no Município de Fortaleza, Ceará, entre 1999 e 2006. Saúde \& Sociedade, v. 19, n. 3, p. 547-56, 2010.

12. Lima KWS, Antunes JLF, Silva ZP. Percepção dos gestores sobre o uso de indicadores nos serviços de saúde. Saúde Soc., v. 4, n. 1, p. 61-71. 2015.

13. Silva LRC. et al. Pesquisa documental: alternativa investigativa na formação docente.[IX Congresso Nacional de Educação/III Encontro Sul Brasileiro de Psicopedagogia, 2009;PUCPR].

14. Bardin L. Análise de conteúdo. Edições 70. Lisboa: LDA, 2009.

15. Shiroma EO. et al. Subsídios teóricos para construção de uma metodologia para análisede documentos de Política Educacional. In. Dossiê: uma metodologia para análise conceitual dedocumentos sobre política educacional, 2004.

16. Brasil. Ministério da Saúde. Secretaria de Atenção à Saúde. Departamento de Atenção Básica. Coordenação Nacional de Saúde Bucal. Projeto SB Brasil 2003: condições de saúde bucal da população brasileira 2002-2003: resultados principais. Brasília: Ministério da Saúde; 2004.

17. Bordin D, Fadel CB. Pacto pela saúde no Brasil: uma análise descritiva da progressão dos indicadores de saúde bucal. Revista Odontol UNESP., v. 41, n. 5, p. 305-11, 2012.

18. Brasil. Ministério da Saúde. Gabinete do Ministro. Comissão Intergestores Tripartite. Resolução n. 2 de 16 de agosto de 2016. Dispõe sobre os indicadores para o processo nacional de pactuação interfederativa, relativo ao ano de 2016. Diário Oficial da União, Brasília (DF), 2016 
out 29 Seção 1.

19. Marques AB. et al. Sistemas de Informação como ferramenta de monitoramento das ações de saúde bucal na Estratégia Saúde da Família da região metropolitana de Curitiba-PR. Revista Bras. Pesq. Saúde, v. 16, n. 1, p. 82-89, 2014.

20. Brasil. Ministério da Saúde. Secretaria de Atenção à Saúde. Departamento de Atenção Básica. A saúde bucal no Sistema Único de Saúde. Brasília: MS, 2018.

21. Brasil. Ministério da Saúde. Gabinete do Ministro. Portaria n. 4.279, 30 de dezembro de 2010. Estabelece diretrizes para a organização da Rede de Atenção à Saúde no âmbito do Sistema Único de Saúde (SUS). Diário Oficial da União, Poder Executivo, Brasília, DF, 30 dez. 2010.

22. Brasil. Ministério da Saúde. Portaria n. 2.669, de 3 de novembro de 2009. Estabelece as prioridades, objetivos, metas e indicadores de monitoramento e avaliação do Pacto pela Saúde, nos componentes pela Vida e de Gestão, e as orientações, prazos e diretrizes do seu processo de pactuação para o biênio 2010 - 2011.Diário Oficial da União, Poder Executivo, Brasília, DF, 03nov. 2009.

23. Brasil. Ministério da Saúde. Portaria n. 3.840, de 12 de dezembro de 2010. Inclui a Saúde Bucal no Monitoramento e Avaliação do Pacto pela Saúde, e estabelece as diretrizes, orientações e prazos do processo de ajuste de metas para o ano de 2011.Diário Oficial da União, Poder Executivo, Brasília, DF, 07dez. 2010.

24. Brasil. Decreto n. 7.508 de 28 de junho de 2011. Regulamenta a Lei $n^{\circ} 8.080$, de 19 de setembro de 1990, para dispor sobre a organização do Sistema Único de Saúde - SUS, o planejamento da saúde, a assistência à saúde e a articulação interfederativa, e dá outras providências.Diário Oficial da União, Poder Executivo, Brasília, DF, 28jun. 2011.

25. Brasil. Ministério da Saúde. Portaria n. 1.580 de julho de 2012. Afasta a exigência de adesão ao Pacto pela Saúde ou assinatura do Termo de Compromisso de Gestão, de que trata a Portaria $n^{\circ} 399 / \mathrm{GM} / \mathrm{MS}$, de 22 de fevereiro de 2006, para fins de repasse de recursos financeiros pelo Ministério da Saúde a Estados, Distrito Federal e Municípios e revoga Portarias.Diário Oficial da União, Poder Executivo, Brasília, DF, 19jul. 2012.

26. França MASA.et al. Oral health indicators in the Interfederative Pacts of the Unified Health System: development in the 1998-2016 period. Revista Odontol UNESP, v. 47, n. 1, p.18$24,2018$. 
$75 / /$

27. Brasil. Ministério da Saúde. Programa Nacional de Melhoria do Acesso e da Qualidade da Atenção Básica (PMAQ): manual instrutivo. Brasília: Ministério da Saúde, 2012. 80p.

28. Fernandes LSP, Peres MA. Associação entre atenção básica em saúde bucal e indicadores socioeconômicos municipais. Revista de Saúde Pública, v. 39, n. 6, p. 930-936, 2005.

29. Loivos C. et al. Acesso às ações de saúde bucal versus indicadores do SIAB/SIASUS: um diálogo possível e necessário. Revista Brasileira Odontolologia., v. 66. p. 263-9, 2009. A Uni

Artigo apresentado em outubro de 2019 Artigo aprovado em janeiro de 2020 Artigo publicado em junho 2020 